\title{
Gezielte Beeinflussung umformtechnisch induzierter Eigenspannungen beim Drahtziehen
}

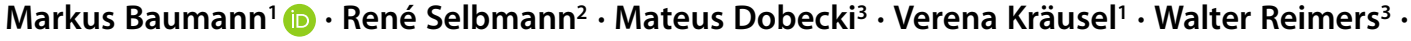 \\ Markus Bergmann²
}

Eingegangen: 15. Januar 2021 / Angenommen: 7. Juni 2021 / Online publiziert: 14. Juli 2021

(c) Der/die Autor(en) 2021

\section{Zusammenfassung}

Nach dem Drahtziehen treten hohe Zugeigenspannungen in den oberflächennahen Schichten des Drahtes auf. Dieser Eigenspannungszustand ist für die weitere umformtechnische Verarbeitung, z. B. für das Biegen zur Herstellung von Torsionstabfedern, ungeeignet. Nach derzeitigen Standards wird dem Drahtziehen eine Wärmebehandlung nachgeschaltet. In diesem Artikel wird ein neuer Ansatz zur Einstellung des Eigenspannungszustandes direkt im Umformprozess des Drahtes vorgestellt. Durch eine Modifikation der Ziehwerkzeuge mit integrierten Umformelementen sollen niedrigere Zugspannungsniveaus in den oberflächennahen Schichten des Drahtes erzeugt werden. Diese Elemente haben wesentlichen Einfluss auf den Grad der Kaltverfestigung, ohne den Drahtdurchmesser zu verändern. Zunächst werden mittels FE-Simulationen verschiedene Werkzeuggeometrien hinsichtlich ihrer Wirkung auf den Umformgrad sowie den Eigenspannungszustand des gezogenen Drahtes analysiert und bewertet. Durch den Einsatz eines speziell konzipierten Drahtziehwerkzeugs werden experimentelle Versuche durchgeführt und Drahtproben erzeugt. Weiterhin wird die Vorgehensweise für die Messung der Eigenspannungen durch den Einsatz von Röntgenbeugung erläutert. Für weiterführende experimentelle Untersuchungen zum Biegen erfolgte die Konzipierung eines modularen Werkzeuges, das eine Variation der Biegeradien und Drahtdurchmesser ermöglicht.

Availability of data and material Not applicable

Code availability Not applicable

Markus Baumann

markus.baumann@mb.tu-chemnitz.de

Institut für Werkzeugmaschinen und Produktionsprozesse, Technische Universität Chemnitz, 09126 Chemnitz, Deutschland

2 Fraunhofer Institut für Werkzeugmaschinen und Umformtechnik, 09126 Chemnitz, Deutschland

3 Institut für Werkstoffwissenschaften und -technologien, Technische Universität Berlin, 10587 Berlin, Deutschland 


\title{
Specific influencing of forming-induced residual stresses during wire drawing
}

\begin{abstract}
After wire drawing high tensile residual stresses occur on the near-surface layers. This residual stress state is unsuitable for further forming processing, e.g. for bending to produce torsion bar springs. In precent industrial processes subsequent process steps, e.g. heat treatment are applied. In this study, a new approach and concept to adjust the residual stress state directly in the forming process of the wire is presented. Lower tensile stress levels in the near-surface layers of the wire shall be generated by modifying the drawing tools with integrated forming elements. These elements have a significant influence on the degree of work hardening without influencing the wire diameter. First, different die geometries are analysed and evaluated by means of FE simulations with regard to their effect on the plastic strain as well as the residual stress state of the drawn wire. By using a specially designed wire drawing tool, experimental tests are performed and wire samples are generated. Furthermore, the procedure for measuring residual stresses by using X-ray diffraction is explained. For further experimental studies of bending, a modular tool was designed that enables the bending radii and wire diameters to be varied.
\end{abstract}

\section{Einleitung}

Die Herausforderung der Massereduktion bei Gewährleistung von gleichen oder sogar besseren mechanischen Eigenschaften bedingt eine technologische Weiterentwicklung bekannter Fertigungsverfahren. So lässt sich beispielsweise durch eine gezielte lokale Einbringung von Druckeigenspannungen die statische und dynamische Festigkeit von Bauteilen steigern. Weitere relevante Aspekte zur Optimierung von Bauteilen sind die Eigenschaftsverbesserung im Betrieb der Komponenten sowie die steigenden Anforderungen bezüglich zur Verfügung stehenden Bauraum und Größe der Bauteile. Dies bedeutet z.B. bei der Herstellung von Torsionsstabfedern die Realisierung kleiner Biegeradien, die mit konventionell hergestellten Federdrähten derzeit nicht versagensfrei gebogen werden können. Basis des Verfahrens zur Modifikation der Eigenspannungszustände beim Drahtziehen bildet das Gradierungspressen, welches zur Beeinflussung der Korngröße während des Fließpressens entwickelt wurde [1]. Das Gradierungspressen verbindet das Fließpressen mit hochgradig plastischer Umformung (severe plastic deformation - spd) in randnahen Werkstoffbereichen und erzeugt somit einen Eigenschaftsgradienten (u. a. Mikrostruktur) über dem Werkstückquerschnitt [2]. Beim Gradierungspressen weist das Werkzeug zusätzliche Geometrieelemente auf, die durch die Änderungen in der Umformrichtung im Werkstoffvolumen des Oberflächenbereiches eine hochgradige Umformung bewirken sowie damit einhergehende Mikrostrukturmodifikation erzeugen. Die Form oder der Durchmesser nach erfolgter Umformung wird jedoch nicht beeinflusst. Das Prinzip des Gradierungspressens ist in Abb. 1 dargestellt. In diesem Anwendungsfall wurde der Durchmesser in einem Fließpressvorgang von $\mathrm{D}=16 \mathrm{~mm}$ auf $\mathrm{D}=10 \mathrm{~mm}$ in vier Stufen mit einer Durchmesserreduzierung an jedem Element von $1,5 \mathrm{~mm}$ umgesetzt [3].
Das Umformen mit integrierten Geometrieelementen in den Matrizen soll vom Druckumformen auf das Drahtziehen übertragen werden. Das Verfahren zur Herstellung von Drähten, die als Ausgangsmaterial für Torsionsstabfedern dienen, ist dem Zugdruckumformverfahren zuzuordnen. Während des Drahtziehens bilden sich bei der Umformung im Draht über den Querschnitt verschiedene Spannungsverteilungen aus. Nach dem Drahtziehen und des damit verknüpften Abbaus der wirkenden Umformkräfte verbleibt im Draht ein charakteristischer Eigenspannungszustand, der durch Druckeigenspannungen im Kern und Zugeigenspannungen im Randbereich gekennzeichnet ist [4]. Diese Spannungen beeinflussen die Umformeigenschaften in weiteren Umformschritten der Halbzeuge und das mechanische Einsatzverhalten z.B. des Drahtes und dessen Lebensdauer unter Last [5]. In der aktuell praktizierten Prozessfolge zur Herstellung der Torsionsstabfedern, die durch das Drahtziehen mit nachgelagerten Drahtbiegen gekennzeichnet ist, begrenzen diese Eigenspannungen im Speziellen die Umformbarkeit z.B. in Biegeoperationen mit kleinen Biegeradien. Die oberflächennahen Zugeigenspannungen, die aus dem Drahtziehen resultieren, überlagern sich mit den Lastspannungen, die bei der Biegeumformung auftreten. Die Folge ist ein Spannungszustand der zur Schädigung des Drahtes führen kann. In den gegenwärtigen Herstellungsprozessen werden die Zugeigenspannungen nach dem Drahtziehen durch eine gezielte Wärmebehandlung vor dem Biegen abgebaut, um eine Rissbildung während der Biegeumformung zu vermeiden. In Abb. 2a ist das Prinzip des konventionellen Drahtziehens dargestellt. In Abb. 2b sind schematisch Eigenspannungszustände an der Drahtoberfläche nach dem konventionellen Drahtziehen und mit reduzierten Zugeigenspannungen aufgezeigt. Die Überlagerung mit den Biegespannungen zeigt einen reduzierten Zugspannungszustand an der Drahtoberfläche. Dadurch können kritische Biegebereiche, wie in Abb. 2c ersichtlich, abgebildet werden, die konventionell nicht herzustellen wären. 
Abb. 1 Prinzip Gradierungspressen [3]

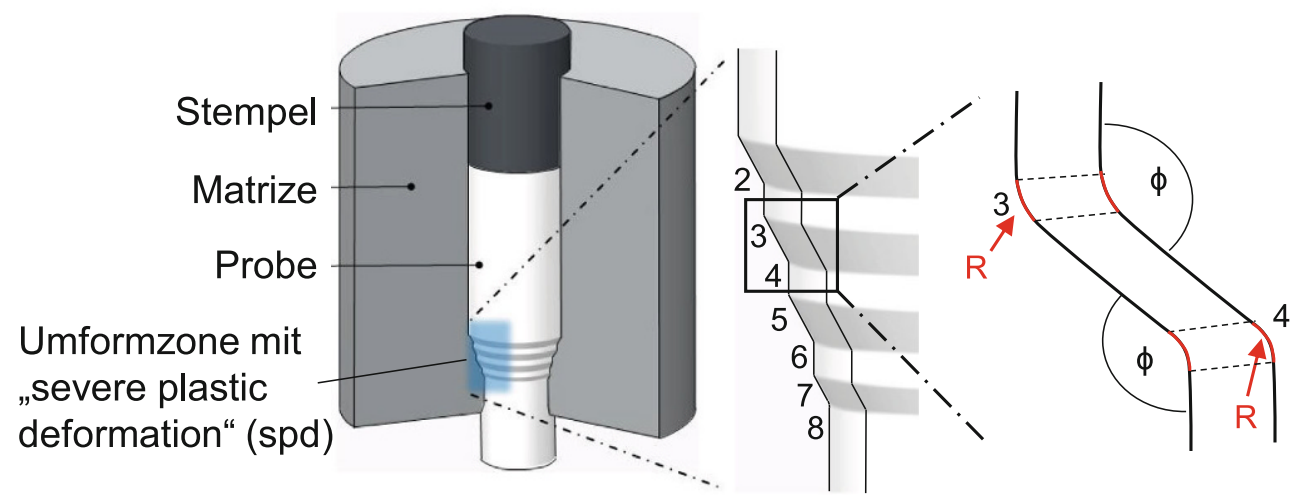

b

Eigenspannungen im Draht nach dem Drahtziehen

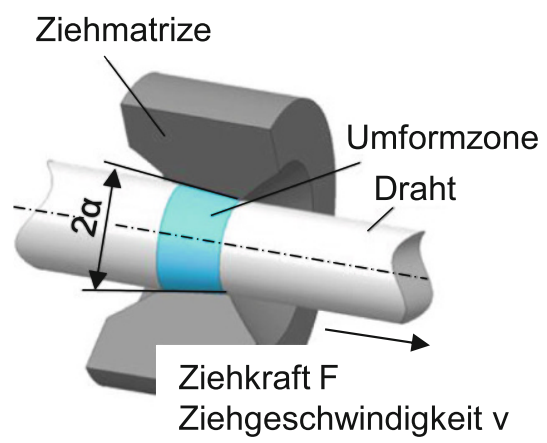

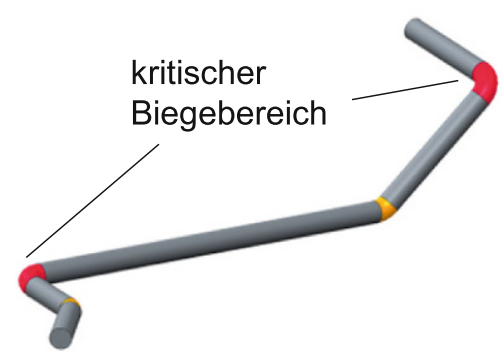

nach dem Drahtziehen

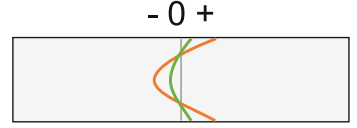

hohe Zugeigenspannungen im Randbereich reduzierte Zugeigenspannungen im Randbereich

Überlagerung der Spannungen im Draht bei der Biegeumformung

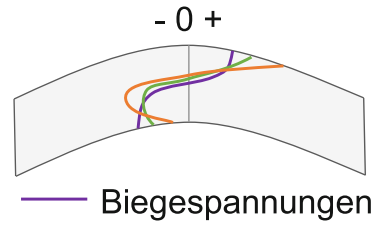

Abb. 2 a Prinzipdarstellung des Drahtziehens b schematische Darstellung der Eigenspannungszustände im Draht und die Überlagerung mit Biegespannungen $\mathbf{c}$ kritischer Bauteilbereich beim Biegen von Torsionsstabfedern

Das Ziel des hier vorgestellten Lösungsansatzes besteht darin, Geometrieelemente zu entwickeln, die in die Werkzeuggeometrie der Ziehmatrize integriert werden, wodurch beim Drahtziehen Eigenspannungszustände erreicht werden, die die Umformbarkeit des Drahts erhöhen. Durch die bei diesem Verfahren bereits beim Drahtziehen umforminduziert reduzierten Zugeigenspannungsniveaus im Randbereich des Drahtes soll die gegenwärtig beim industriellen Prozess notwendige Wärmebehandlung und das Kugelstrahlen substituiert werden. Die statischen und dynamischen Eigenschaften des Drahtes als Ausgangsmaterial sollen durch die gezielte Einstellung der Eigenspannungen beim Drahtziehen optimiert und in Folge dessen auch die Umformung sowie Belastbarkeit der Bauteile - Torsionsstabfedern verbessert werden.

\section{Konzept und Vorgehensweise}

\subsection{Materialcharakterisierung}

Als Material für die Untersuchung wurde der austenitische Edelstahl 1.4301 gewählt. Dieser zeichnet sich durch die Korrosionsbeständigkeit aus und verfestigt stark bei der Kaltumformung und wird in technischen Federn eingesetzt [6]. Um einen definierten Ausgangszustand des Drahtes für die grundlegenden experimentellen Untersuchungen, mit geringer Vorbelastungen im Gefüge sowie einen reduzierten Eigenspannungszustand des Stabmaterials zu generieren, erfolgte zunächst die Ermittlung einer geeigneten Wärmebehandlung. Das Lösungsglühen des Ausgangsmaterials von $15 \mathrm{~min}$ (+6 min Durchheizzeit) bei $1050^{\circ} \mathrm{C}$ und anschließender Abkühlung an ruhender Luft konnte als zielführend ausgewählt werden, um die Eigenspannungen im Ausgangsmaterial betragsmäßig zu reduzieren. Nach der Wärmebehandlung beträgt die mittlere Korngröße $28 \pm 9 \mu \mathrm{m}$ 
Abb. 3 a Fließkurven ohne Approximation für verschiedene Prüftemperaturen und Dehnraten b Approximation der Fließkurve für höhere Dehnraten mit den ermittelten Parametern für den Fließkurvenansatz nach Swift
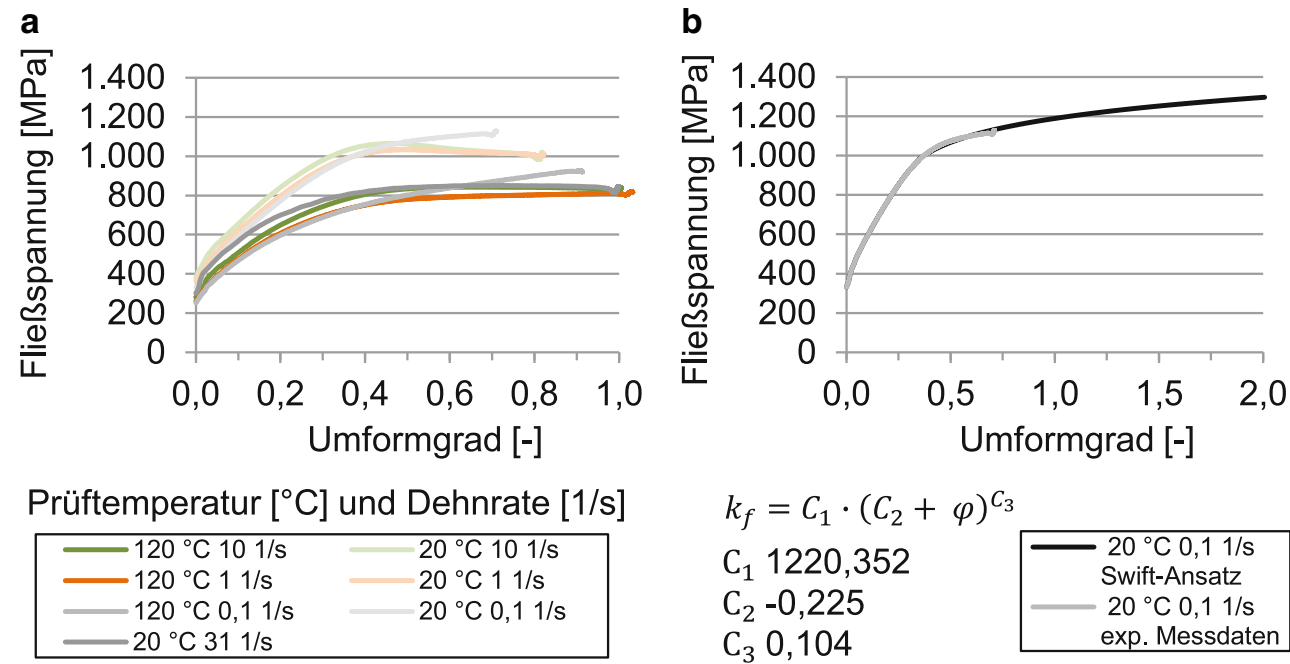

$k_{f}=C_{1} \cdot\left(C_{2}+\varphi\right)^{C_{3}}$
$\mathrm{C}_{1} 1220,352$
$\mathrm{C}_{2}-0,225$
$\mathrm{C}_{3} 0,104$$\quad \begin{array}{r}20^{\circ} \mathrm{C} 0,11 / \mathrm{s} \\ \text { Swift-Ansatz } \\ 20^{\circ} \mathrm{C} 0,11 / \mathrm{s} \\ \text { exp. Messdaten }\end{array}$ bei einer Härte von $164 \pm 18$ HV0,5. Weiterhin wurden in der Materialcharakterisierung Druckversuche mit der Anlage Gleeble System 3800 durchgeführt. Aus den somit erarbeiteten Spannungs-Dehnungskurven bei verschiedenen Temperaturen und Dehnraten wurden für die FE-Simulation Fließkurven (Abb. 3a) ermittelt. Die Temperatur von $120^{\circ} \mathrm{C}$ bildet eine Drahttemperatur in einem industriellen Drahtziehprozess ab. Durch Approximation der Kurvenverläufe erfolgte die Abbildung der Fließkurven für höhere Umformgrade. Für die Extrapolation wurde der Ansatz nach Swift genutzt [7]. Für die Dehnrate von 0,1 1/s ist die Erweiterung des Kurvenverlaufes in Abb. $3 b$ dargestellt. Beginnend von der Fließspannung $\varphi 0,25$ bis zum Ende der experimentellen Messdaten wurde mittels der Methode der kleinsten Fehlerquadrate die Variablen $\mathrm{C} 1, \mathrm{C} 2$ und $\mathrm{C} 3$ für die Extrapolation bestimmt. Die Fließkurve für die Berechnungen in der FE-Simulation setzt sich aus den Messdaten und der Extrapolation zusammen. Bis $\varphi$ 0,35 werden die experimentellen Daten und fortführend die extrapolierten Daten implementiert.

\subsection{FE-Simulation Drahtziehen}

Um eine grundlegende Auswahl an Ziehmatrizen zu erarbeiten und den Einfluss der Geometrieelemente auf den resultierenden Umformgrad zu untersuchen, wurden FE-Modelle im Programm Abaqus CAE (Version 2020) aufgebaut. Für erste grundlegende Analysen des Drahtziehens wurde ein 2D-axialsymmetrischer Modellaufbau mit expliziter Berechnungsmethode, ohne Massenskalierung gewählt. Wenn die Weiterverarbeitung mittels Drahtbiegen betrachtet wird, sind 3D-Modelle erforderlich. Die Phasenumwandlung infolge der verformungsinduzierten Martensitbildung bei dem Material 1.4301 ist derzeit noch nicht Bestandteil des FE-Modells, befindet sich aber in der Entwicklung. In diesem Zusammenhang soll die Validierung der FE-Simu- lationen speziell in der Bewertung der Eigenspannungen verbessert werden. Die Proben wurden vereinfacht mit einem eigenspannungsfreien Ausgangszustand auf der Makroskala definiert. Um das Netz, mit einer Elementkantenlänge von $0,1 \mathrm{~mm}$, bei der Umformung zu entzerren, wurde, statt einem Remeshing, die Abaqus Methode ALE Adaptive Mesh verwendet. Als Reibmodell fand das Reibfaktormodell Anwendung, eine Kombination aus dem Reibmodell nach Coulomb und nach Tresca. Dieses Modell wird genutzt, wenn bei der Umformung zwischen Werkstück, in diesem Fall den Draht, und dem Werkzeug, der Ziehmatrize, hohe Kontaktnormalspannungen auftreten. Dieses tribologische System wird mit dem Reibmodell nach Coulomb nicht exakt abgebildet [8]. Die Reibwerte wurden in Abstimmung mit den benötigten Ziehkräften verglichen und angepasst. Über die Prozessdauer konnte eine Differenz von ca. $10 \%$ ermittelt werden. Die Werkzeuge wurden als Starrkörper modelliert. In Anlehnung an [3, 9] erfolgte eine Durchmesserreduzierung des Ausgangsmaterials bei der Umformung von $\mathrm{D}=12 \mathrm{~mm}$ auf $\mathrm{D}=10,8 \mathrm{~mm}$ in einer Ziehstufe. Für die konventionelle Geometrie wurden der Ziehwinkel $2 \alpha$ und die zylindrische Führung nach $[9,10]$ ausgelegt. Für die Auswertung der resultierenden Eigenspannungen nach der Umformung, schloss sich eine implizite Berechnung des Spannungszustandes mit der Entlastung der jeweiligen Probe an. In Abb. 4 ist der 2D-axialsymmetrische Modellaufbau mit starrer Matrize dargestellt. Die Ziehbewegung wurde über ein Node-Set erzeugt, über dieses auch die benötigte Ziehkraft ausgewertet werden konnte. Die Proben wurden in den FE-Simulationen vollständig durch die spezifischen Ziehmatrizen gezogen.

In der Auslegung der Matrizen war es zunächst in einer ersten Entwicklungsstufe das Ziel, einzelne Geometrieelemente einzubringen und den Ziehwinkel $2 \alpha$ von $12^{\circ}$ der konventionellen Matrize sowie die zylindrische Führung in der Ziehmatrize von $1,64 \mathrm{~mm}$ einheitlich umzusetzen. Ne- 


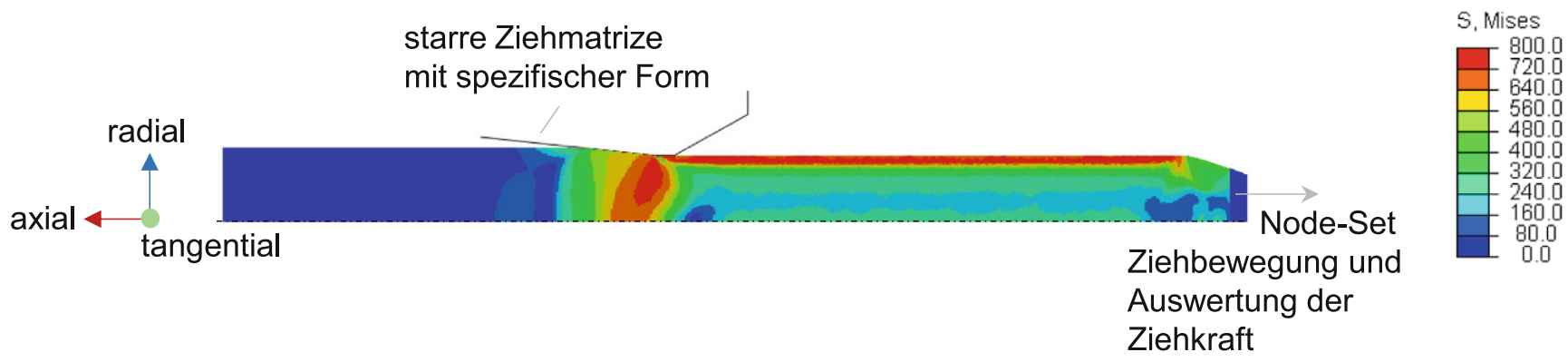

Abb. 4 FE-Simulation Drahtziehen - 2D-axialsymmetrischer Modellaufbau

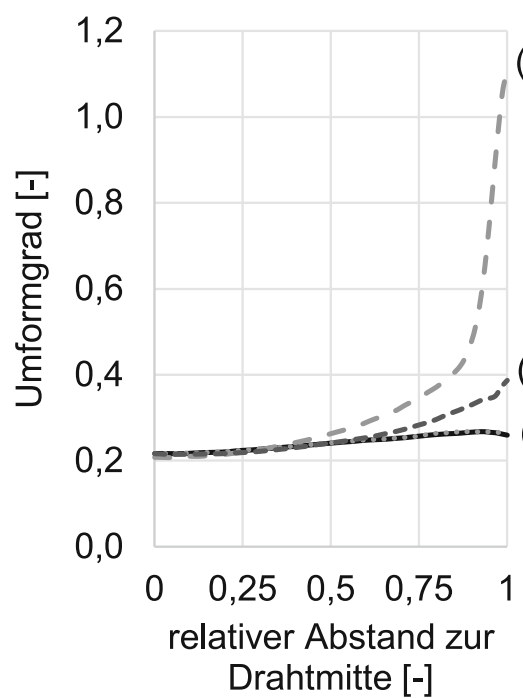

(2)
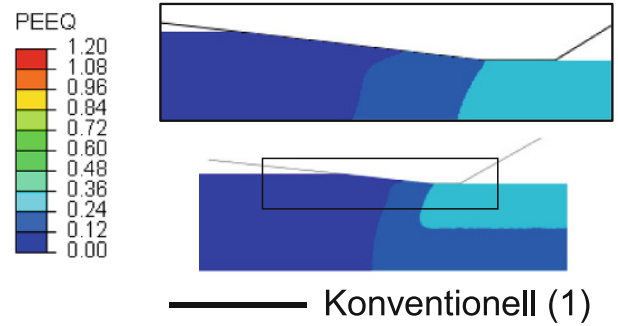

(4)

(1) (3)
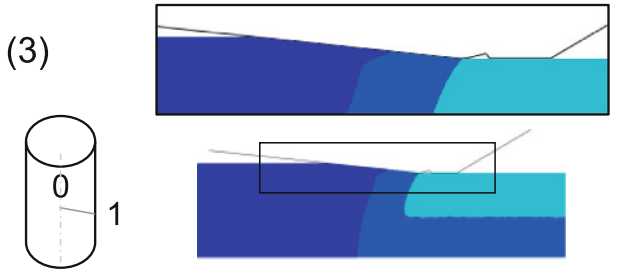

Konkav (3)
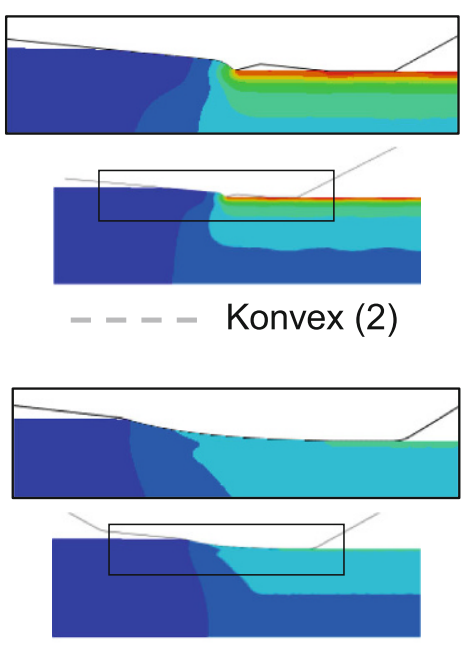

Traktrix (4)

Abb. 5 FE-Simulation Drahtziehen - Umformgrad beim Drahtziehen mit ausgewählten Geometrievarianten

ben der konventionellen Variante wurde ein zusätzlich in die Ziehmatrize integriertes konvexes bzw. konkaves Geometrieelement entwickelt. Das Geometrieelement der konvexen und der konkaven Form weist eine Größe von 0,25 mm auf. Die Winkel und Flächen der Innenkonturen der Ziehmatrizen wurden nach der Fertigung mittels taktiler Messung ermittelt und in das FE-Modell eingearbeitet. Die Vorgehensweise wird im Abschnitt „Experimentelle Untersuchungen“ erläutert. Der Winkel am konvexen Geometrieelement konnte mit $39^{\circ}$ erfasst und im FE-Modell berücksichtigt werden. Eine Traktrix-Form wurde auf Basis positiver Erfahrungen beim Feinschneiden [11] und Tiefziehen [12] in einer zweiten Entwicklungsstufe umgesetzt. Ziel war es, den Umformgrad zu erhöhen, diesen jedoch nicht in Form einer lokalen Beeinflussung, wie beim konvexen Geometrieelement, sondern durch eine kontinuierliche Steigerung zu erzielen. In Abb. 5 sind ausgewählte Geometrievarianten in Form einer konventionellen, konvexen, konkaven und einer Traktrix-Variante aufgezeigt.

In der Auswertung der FE-Simulationen der erzielten axialen Eigenspannungen, welche in Abb. 6 dargestellt sind, wird deutlich, dass die Zugeigenspannung an der Probenoberfläche durch das Ziehen mit der Traktrix-Form gegen- über der konventionellen Variante um ca. 33\% reduziert werden konnte. Diese Matrize wurde jedoch noch nicht im Experiment getestet und befindet sich derzeit noch in der Optimierung, da diese in der zweiten Entwicklungsstufe nach den experimentellen Tests sowie Bewertungen der Varianten konvex und konkav erarbeitet wurde. Es ist zudem in Abb. 5 ersichtlich, dass mit den Formelementen bei der konvexen sowie auch bei der Traktrix-Form ein erhöhter Umformgrad erzielt wird. In den experimentellen Untersuchungen spiegelt sich dieser in einer lokalen Martensitbildung bei der konvexen Variante an der Oberfläche wider. In der Auswertung und Bewertung der erzielten Eigenspannungen werden die axialen Bestandteile betrachtet.

\subsection{Experimentelle Bestimmung der Eigenspannungen}

Eigenspannungen können mithilfe der röntgenografischen Spannungsanalyse (RSA) ortsaufgelöst ermittelt werden. Die röntgenografische Analyse elastischer Spannungen in vielkristallinen Werkstoffen beruht auf der experimentellen Ermittlung von Gitterdehnungen in verschiedenen Messrichtungen $(\varphi, \psi)(\varphi$-Probendrehung, $\psi$-Probenkippung), 
Abb. 6 FE-Simulation Drahtziehen - Axiale Eigenspannungen über den Querschnitt in Abhängigkeit der Geometrievarianten
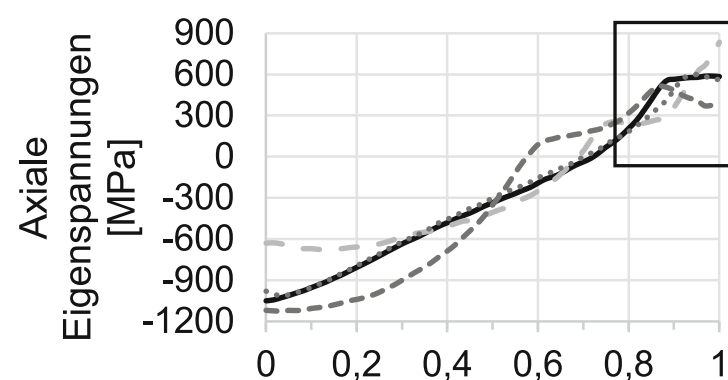

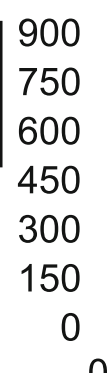

relativer Abstand zur Drahtmitte [-]

aus denen mittels geeigneter Transformationsbeziehungen sowie des Hooke'schen Gesetzes die Eigenspannungen berechnet werden. Grundlage der Dehnungsanalyse bildet die Ermittlung der Netzebenenabstände d(hkl) (hkl-Miller'sche Indizes) gemäß der Bragg'schen Gleichung:

$\mathrm{n} \lambda=2 \mathrm{~d}(\mathrm{hkl}) \sin \theta$

worin $\lambda$ die Wellenlänge der Strahlung, $n$ die Beugungsordnung der Interferenz hkl und $\theta$ deren Bragg-Winkel beschreibt. Für die ortsaufgelöste Eigenspannungsanalyse im Reflexionsmodus werden Lage und Ausdehnung des Messvolumens durch die Anordnung von Blenden im Primärund Sekundärstrahl und durch den eingestellten Beugungswinkel festgelegt. Die Bestimmung der oberflächenparallelen Eigenspannungen mithilfe röntgenografisch ermittelter Gitterdehnungen an den Drahtproben erfolgte mit der $\sin ^{2}$ $\Psi$-Methode [13].

Die Durchführung der winkeldispersiven Röntgenbeugung wurde mit monochromatischer $\mathrm{Co}-\mathrm{K} \alpha$-Strahlung $(\lambda=0,178897 \mathrm{~nm}$, Eindringtiefe gemittelt $\tau=8 \mu \mathrm{m})$ unter Verwendung eines 4-Kreis $\psi$ Diffraktometers der Firma Huber umgesetzt. In Abb. 7 ist die Einspannung der Drahtprobe in das Diffraktometer aufgezeigt.

Für die Bestimmung der Eigenspannungen wurde der Austenit-311-Reflex und der $\alpha^{\prime}$-Martensit-211-Reflex gewählt, da diese neben einer hohen Intensität im Rückstrahlbereich (Linienlage $2 \theta=111,4^{\circ}$ bzw. $99,7^{\circ}$ ), geringere Beeinflussungen von Textureffekten aufweisen und

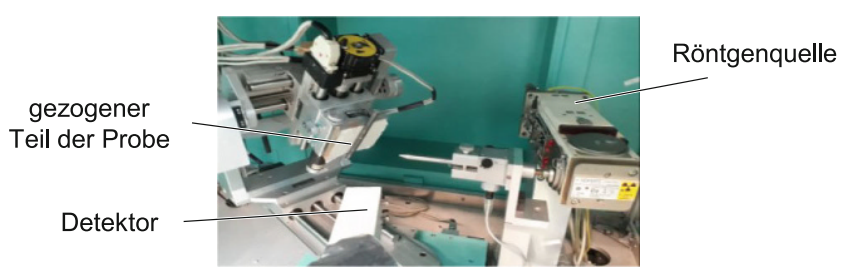

Abb. 7 Messaufbau Röntgenbeugung aufgrund der Multiplizität repräsentativ für den vorliegenden Eigenspannungszustand im untersuchten Messvolumen sind. Die experimentelle Durchführung der $\sin ^{2} \Psi$-Methode bestand in der Aufnahme des Austenit-311-Reflexes und des $\alpha$ '-Martensit-211-Reflexes für neun verschiedene $\Psi$-Kippungen $\left( \pm 63,435^{\circ}, \pm 50,787^{\circ}, \pm 39,232^{\circ}, \pm 26,565^{\circ}\right.$ und $0^{\circ}$ ). Für die Auswertung der Eigenspannungen wurden die diffraktionselastischen Konstanten $\mathrm{s}_{1}=-1,77 \cdot 10^{-6} \mathrm{MPa}$; $1 / 2 \mathrm{~s}_{2}=7,11 \cdot 10^{-6} \mathrm{MPa}$ (Austenit) und $\mathrm{s}_{1}=-1,27 \cdot 10^{-6} \mathrm{MPa}$; $1 / 2 \mathrm{~s}_{2}=5,8 \cdot 10^{-6} \mathrm{MPa}$ ( $\alpha^{\prime}$-Martensit) verwendet. Zur Eingrenzung des Messvolumens wurde ein Rundkollimator mit einem Durchmesser von $2 \mathrm{~mm}$ verwendet und der Winkelbereich $2 \theta$ von $108^{\circ}-14^{\circ}$ (Austenit) bzw. $96^{\circ}-103^{\circ}$ ( $\alpha^{\prime}$-Martensit) mit einer Schrittweite von $\Delta 2 \theta=0,05^{\circ}$ und einer Zählzeit von $25 \mathrm{~s}$ pro Schritt im $\Omega$-20-Modus gemessen. Der Streuuntergrund wurde mit einem $0,4^{\circ}$ Soller und einem nachgeschalteten (001)-LiF-Analysator verringert.

Die Vorteile dieses zerstörungsfreien Verfahrens liegen in der Unempfindlichkeit gegenüber Unsicherheiten des dehnungs- bzw. spannungsfreien Gitterparameters $d_{0}$, die Bestimmung eines Eigenspannungswertes basiert auf vielen Messwerten und eine Abschätzung der Messunsicherheit anhand von Fehlergrenzen ist mithilfe statistischer Gesetze gegeben. Für das $\sin ^{2} \Psi$-Verfahren ist demzufolge keine röntgenographische Ermittlung absoluter Linienlagen erforderlich, sondern es genügt, Linienlagenverschiebungen in Abhängigkeit von $\sin ^{2} \Psi$ zu ermitteln.

\subsection{Experimentelle Untersuchungen}

\subsubsection{Drahtziehvorrichtung und experimentelle Versuche}

Für die experimentelle Umsetzung des Lösungsansatzes wurde eine Ziehvorrichtung entwickelt (Abb. 8, rechts). In der Versuchsvorrichtung werden die Proben senkrecht eingespannt und die Ziehmatrize wird nach oben über die Probe gezogen. Damit wird eine homogene Verteilung des Schmierstoffes (Ziehöl oder Ziehseife) ermöglicht. Um 
Abb. 8 Drahtziehvorrichtung und Versuchsaufbau in der Servopresse sowie in der Hochgeschwindigkeitsprüfmaschine
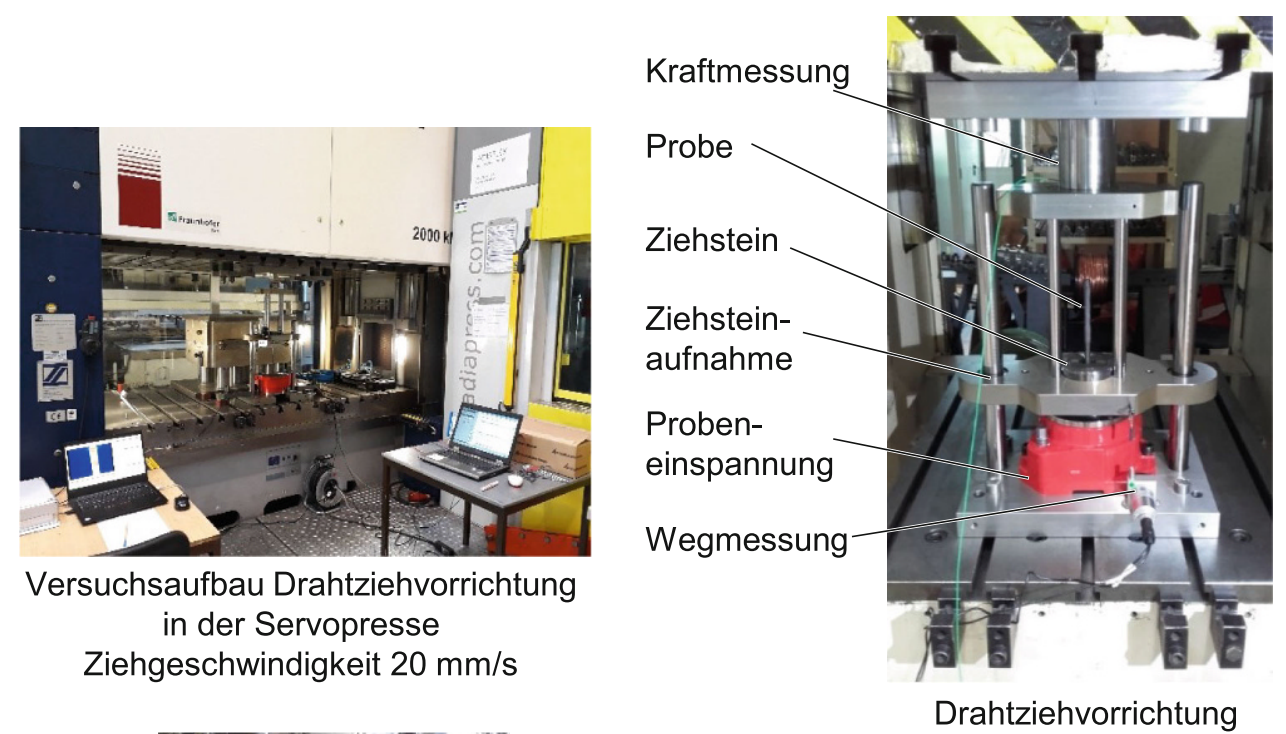
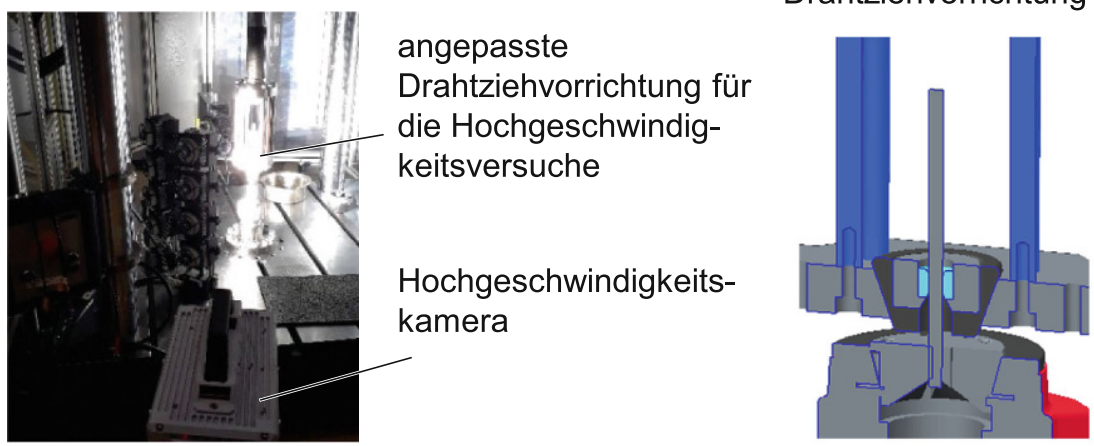

Versuchsaufbau Drahtziehvorrichtung in der Hochgeschwindigkeitsprüfmaschine Ziehgeschwindigkeit 2000 mm/s

Schnittdarstellung Probeneinspannung und Ziehsteinaufnahme verschiedene Geometrien der Ziehmatrizen zu untersuchen, ist die Ziehmatrize in der Ziehmatrizenaufnahme austauschbar. Die Matrizen wurden auf Basis der Untersuchungen in den FE-Simulationen entwickelt und gefertigt. Mittels taktilen Messverfahren wurden nach der Fertigung die Schnitte der Innengeometrien der Matrizen in $0^{\circ}$, $90^{\circ}, 180^{\circ}$ und $270^{\circ}$ bezogen auf den Umfang gemessen. Die festgestellten Abweichungen wurden in die FE-Modelle eingearbeitet und für die weitere Optimierung mit beachtet. Die Drahtziehvorrichtung ist in verschiedenen Umformmaschinen flexibel einsetzbar. Ebenso verfügt die Vorrichtung über eine integrierte Weg- (Seilzugsensor) und Kraftmessung (Kistler Kraftmessdose). Die eingesetzten Proben haben einen Ausgangsdurchmesser von $\mathrm{D}=12 \mathrm{~mm}$ und eine Länge von $\mathrm{L}=100 \mathrm{~mm}$. Nach dem bereits beschriebenen Glühvorgang und dem folgenden Abkühlen an Luft werden diese in die Vorrichtung eingespannt und auf einen Durchmesser von $\mathrm{D}=10,8 \mathrm{~mm}$ und eine Länge von $\mathrm{L}=125 \mathrm{~mm}$ gezogen. Erste Versuche erfolgten zunächst in einer Servopresse (Abb. 8, links oben). Dabei wurde die Vorrichtung fest mit dem Pressentisch verbunden. Die
Aufbringung der Ziehkraft erfolgte über den Rückhub des Pressenstößels. Der Vorteil dieses Betriebes ist mit einem definierten Geschwindigkeitsprofil zu begründen. Die Bewegungsgeschwindigkeit betrug $20 \mathrm{~mm} / \mathrm{s}$. Um die Ziehgeschwindigkeit dem Drahtziehen auf industriellen Anlagen anzunähern, fanden weiterführende Versuche auf einer Hochgeschwindigkeitsprüfanlage (Abb. 8, links unten) statt. Die Ziehgeschwindigkeit konnte auf $2000 \mathrm{~mm} / \mathrm{s}$ gesteigert werden. Durch die Variation der Matrizengeometrie besteht die Möglichkeit, Einfluss auf die plastische Umformung (Abb. 5) und damit auf die resultierenden Eigenspannungszustände zu nehmen.

Die Modifikation der oberflächennahen Eigenspannungen hinsichtlich des Abbaus von Zugeigenspannungen zeigt sich in der Verwendung konvexer und konkaver Matrizen im Vergleich zur konventionellen Matrize während des Ziehvorgangs. So betragen die gemittelten Eigenspannungen an der Probenoberfläche in axialer Richtung unter Verwendung der konventionellen Matrize $\sigma=+135 \pm 25 \mathrm{MPa}$, wohingegen die konvexe bzw. konkave Matrizengeometrie Eigenspannungen von $\sigma=+106 \pm 23 \mathrm{MPa}$ bzw. $\sigma=+80 \pm 31 \mathrm{MPa}$ 
Abb. 9 Biegewerkzeug mit den Werkzeugvarianten Abkanten und 3-Punkt-Biegen

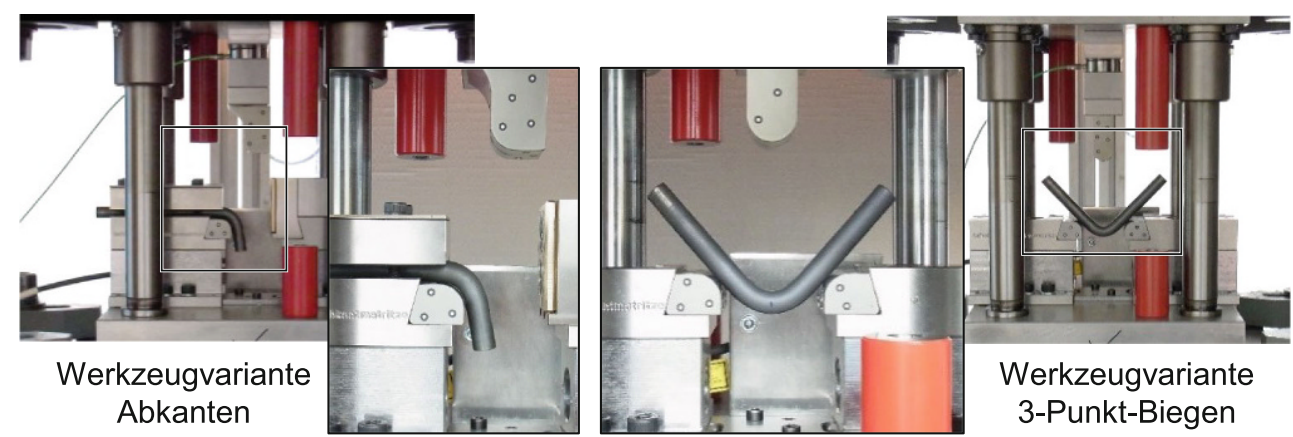

aufweisen, was einer Reduzierung der Zugeigenspannungen um bis zu $40 \%$ entspricht. In Anbetracht sich anschließender Biegeumformungen und der Realisierung kleiner Biegeradien besteht mit der gezielten Anpassung der Matrizen damit die Möglichkeit, den vorliegenden oberflächennahen Eigenspannungszustand der gezogenen Produkte weiter zu optimieren.

\subsubsection{Biegevorrichtung}

Um in weiteren geplanten experimentellen Untersuchungen die gezogenen Proben hinsichtlich der Biegeumformung zu überprüfen, wurde bereits eine Biegevorrichtung entwickelt. Dabei bestand das Ziel darin, neben den speziell bei der Herstellung von Torsionsstabfedern vorherrschenden Drei-Punkt-Biegung und dem Abkanten die Prozesskräfte bei der Umformung mit aufzuzeichnen. In Abb. 9 ist das Biegewerkzeug mit den Varianten Abkanten und Drei-Punkt-Biegung aufgezeigt. Mittels einer integrierten Kraftmessdose (Kistler) als auch einen Laser-Triangulations-Wegsensor (Micro-Epsilon) können die Prozessdaten Kraft und Weg für den Abgleich mit der FE-Simulation aufgezeichnet werden. Die Biegeradien werden durch den Austausch der Aktivelemente angepasst, wodurch die Einflüsse verschiedener Eigenspannungszustände der Drähte auf das Biegeverhalten, im Speziellen auf die möglichen Biegeradien untersucht werden sollen. Umfangreiche Experimente stehen in diesem Zusammenhang noch aus, wodurch die Ergebnisse noch nicht aufgeführt werden können.

\section{Zusammenfassung und Ausblick}

Der Lösungsansatz, Ziehmatrizen mit integrierten Geometrieelementen in der FE-Simulation zu entwickeln, in den experimentellen Untersuchungen den Drahtziehprozess mit eigens konstruierten Umformwerkzeugen zu überprüfen und die Eigenspannungen durch Röntgenbeugung zu ermitteln, wurde vorgestellt. Im Rahmen der bisherigen Untersuchungen wurde der Umformprozess des Drahtziehens, im Zusammenhang mit dem eingesetzten Material 1.4301 untersucht. Primär wurden vertiefte Erkenntnisse zu umformtechnisch induzierten Eigenspannungen erarbeitet und die Einstellung von Eigenspannungszuständen im umgeformten Halbzeug analysiert. Auf Basis dieser Ergebnisse und des Konzeptes besteht nun die Möglichkeit, die induzieren Eigenspannung gezielt durch eine Modifikation des Umformprozesses beim Drahtziehen zu beeinflussen. Dies konnte in ersten Versuchen bereits nachgewiesen werden.

Im weiteren Verlauf der Untersuchungen wird nun schrittweise ein modifizierter Drahtziehprozess ausgelegt. Dabei liegt der Fokus auf der weiteren Reduzierung der Zugeigenspannungen im Randbereich des Drahtes. Die dadurch begünstigten Eigenschaften des Halbzeuges sollen auf die Weiterverarbeitung verifiziert werden. Die für Drahtprodukte charakteristischen Biegeoperationen soll dabei in ihren Prozessgrenzen erweitert werden. Das Werkzeug wurde bereits konzipiert und vorgestellt. Ziele sind geringere Biegeradien, geringere Neigung zur Rissbildung und mechanisch günstigere Bauteileigenschaften einzustellen. Durch dieses Vorgehen sollen kostenintensive Wärmeund Nachbehandlungsprozesse substituiert werden.

Danksagung Die Autoren bedanken sich bei der Deutschen Forschungsgemeinschaft (DFG) für die finanzielle Unterstützung der Forschungsarbeit im Rahmen des Schwerpunktprogramms „SPP2013“ unter den Fördernummern SPP 2013 BE 5983/2-2; KR 4682/5-2; RE 688/ $75-2$

Funding Statement This study was funded by the Deutsche Forschungsgemeinschaft (DFG, German Research Foundation) of the priority program - SPP 2013 BE 5983/2-2; KR 4682/5-2; RE 688/752

Author Contribution Not applicable

Funding Open Access funding enabled and organized by Projekt DEAL.

Interessenkonflikt M. Baumann, R. Selbmann, M. Dobecki, V. Kräusel, W. Reimers und M. Bergmann geben an, dass kein Interessenkonflikt besteht.

Open Access Dieser Artikel wird unter der Creative Commons Namensnennung 4.0 International Lizenz veröffentlicht, welche die Nutzung, Vervielfältigung, Bearbeitung, Verbreitung und Wiedergabe in jeglichem Medium und Format erlaubt, sofern Sie den/die ursprüng- 
lichen Autor(en) und die Quelle ordnungsgemäß nennen, einen Link zur Creative Commons Lizenz beifügen und angeben, ob Änderungen vorgenommen wurden.

Die in diesem Artikel enthaltenen Bilder und sonstiges Drittmaterial unterliegen ebenfalls der genannten Creative Commons Lizenz, sofern sich aus der Abbildungslegende nichts anderes ergibt. Sofern das betreffende Material nicht unter der genannten Creative Commons Lizenz steht und die betreffende Handlung nicht nach gesetzlichen Vorschriften erlaubt ist, ist für die oben aufgeführten Weiterverwendungen des Materials die Einwilligung des jeweiligen Rechteinhabers einzuholen.

Weitere Details zur Lizenz entnehmen Sie bitte der Lizenzinformation auf http://creativecommons.org/licenses/by/4.0/deed.de.

\section{Literatur}

1. Zachäus R, Bergmann M (2013) Verfahren und Vorrichtung zur Korngrößenbeeinflussung eines Werkstückes sowie Werkstück. Deutsches Patent (DE102012006952B4)

2. Bergmann M, Sterzing A, Landgrebe D (2015) Influencing the gradient of material properties by gradation extrusion. Appl Mech Mater 794:166-173

3. Landgrebe D, Sterzing A, Schubert N, Bergmann M (2016) Influence of die geometry on performance in gradation extrusion using numerical simulation and analytical calculation. CIRP Ann Manuf Technol 65:269-272. https://doi.org/10.1016/j.cirp.2016.04.128
4. Atienza JM, Ruiz-Hervias J, Martinez-Perez ML, Mompean FJ, Garcia-Hernandez M, Elices M (2005) Residual stresses in cold drawn pearlitic rods. Scr Mater 52(12):1223-1228

5. He S, Van Bael A, Li SY, Van Houtte P, Mei F, Sarban A (2003) Residual stress determination in cold drawn steel wire by FEM simulation and X-ray diffraction. Mater Sci Eng A 346(1-2):101-107

6. Schatt W, Simmchen E, Zouhar G (1998) Konstruktionswerkstoffe des Maschinen- und Anlagenbaus, 5. Aufl. Deutscher Verlag für Grundstoffindustrie, Stuttgart

7. Venet G, Balan T, Baudouin C, Bigot R (2018) Direct usage of the wire drawing process for large strain parameter identification. Int $\mathrm{J}$ Mater Form 12(5):875-888

8. Schmid SR, Saha PK, Wang J, Schmitz T (2020) Developments in tribology of manufacturing processes. J Manuf Sci Eng. https://doi. org/10.1115/1.4047723

9. Tschätsch H (2005) Praxis der Umformtechnik: Arbeitsverfahren, Maschinen, Werkzeuge. Vieweg, Wiesbaden

10. Schneider F, Lang G (1973) Stahldraht - Herstellung und Anwendung. VEB Deutscher Verlag für Grundstoffindustrie, Leipzig

11. Hoffmann H, Fritsch C, Thomas V, Schirmacher F (2002) Feinschneiden und Umformen von Dickblech aus Aluminiumlegierungen. EFB-Forschungsbericht. Europäische Forschungsgemeinschaft für Blechbearbeitung e. V.,

12. Hellwig W (2009) Spanlose Fertigung: Stanzen. Vieweg+Teubner, Wiesbaden

13. Macherauch E, Müller P (1961) Das $\sin ^{2} \Psi$ Verfahren von röntgenographische Eigenspannungen. Z Angew Phys 13:305-312 